\title{
Impact of Personal Protective Equipment on the Performance of Emergency Pediatric Procedures by Prehospital Providers
}

Maybelle Kou, MD, MEd; Aaron J. Donoghue, MD, MSCE; Helen Stacks, DNP; Adam Kochman, MD; Meghan Semião, BS; Mark Nash; David Siegel, MD; Lawrence Ku, MD; Julie Debski, BS; Jia-Yuh Chen, PhD; Gaurav Sharma, PhD; Leigh Gosnell, BA; Steven Krug, MD; Mark D. Adler, MD; on behalf of the Best Pharmaceuticals for Children Act - Pediatric Trials Network

\section{ABSTRACT}

Background: Personal protective equipment (PPE) is worn by prehospital providers (PHPs) for protection from hazardous exposures. Evidence regarding the ability of PHPs to perform resuscitation procedures has been described in adult but not pediatric models. This study examined the effects of PPE on the ability of PHPs to perform resuscitation procedures on pediatric patients.

Methods: This prospective study was conducted at a US simulation center. Paramedics wore normal attire at the baseline session and donned full Level B PPE for the second session. During each session, they performed timed sets of psychomotor tasks simulating clinical care of a critically ill pediatric patient. The difference in time to completion between baseline and PPE sessions per task was examined using Wilcoxon signed-rank tests.

Results: A total of 50 paramedics completed both sessions. Median times for task completion at the PPE sessions increased significantly from baseline for several procedures: tracheal intubation $(+4.5 \mathrm{~s} ; P=0.01)$, automated external defibrillator (AED) placement $(+9.5 \mathrm{~s} ; P=0.01)$, intraosseous line insertion ( $+7 \mathrm{~s} ; P<0.0001)$, tourniquet $(+8.5 \mathrm{~s} ; P<0.0001)$, intramuscular injection $(+21-23 \mathrm{~s}$, $P<0.0001)$, and pulse oximetry $(+4 \mathrm{~s} ; P<0.0001)$. There was no significant increase in completion time for bag-mask ventilation or autoinjector use.

Conclusions: PPE did not have a significant impact on PHPs performing critical tasks while caring for a pediatric patient with a highly infectious or chemical exposure. This information may guide PHPs faced with the situation of resuscitating children while wearing Level B PPE.

Key Words: pediatric patients, personal protective equipment, prehospital providers

$\mathrm{T}$ he 2015 Ebola virus epidemic in West Africa renewed worldwide attention on the ability of health-care entities to handle highly infectious patients. Development and implementation of hospital protocols to prepare for Ebola and other exposures requiring high-level personal protective equipment (PPE) occurred throughout the United States and worldwide. ${ }^{1,2}$ These efforts included training health-care providers (HCPs) to care for infected HCPs being evacuated for treatment in their native countries. Following chemical attacks in the United Kingdom and Syria, attention has also increased on the protection of HCPs in scenarios involving mass poisonings. In 2020, these preparations now include protecting HCPs from the novel coronavirus.

PPE refers to barrier clothing, gloves, and/or headgear designed to protect an individual from exposure to biologic, chemical, radiological, or environmental hazards in the prehospital or hospital environment. The key focus of PPE in health care is to provide a barrier for muco-cutaneous and/or respiratory protection, with a wide spectrum of equipment. The selection of the appropriate level of PPE for HCPs is based on the specific biohazard and environment in which the patient is treated, and for which an appropriate combination of respiratory and/or chemical protection should be anticipated, classified as Levels A through D from most to least protective. ${ }^{3}$

As front-line medical professionals, prehospital providers (PHPs) have an important role to safely care for patients with potentially transmissible illness. When the identity of a prehospital hazardous material is unknown, fully encapsulating nonpermeable, gastight protective gear (Level A) is recommended. ${ }^{4}$ Level B PPE is visually similar to Level A gear, but used for the highest degrees of respiratory protection when a 
substance is known. Both of these forms of PPE are bulky, limit tactile and auditory feedback, and restrict mobility. ${ }^{5}$ A few publications on adult resuscitation describe the decreased ability of providers to perform resuscitation procedures in PPE,${ }^{6,7}$ but research on the impact of PPE on procedural performance by PHPs caring for children remains limited. It is also unknown as to whether or how specific elements of care delivery should be modified when providers must wear PPE to resuscitate pediatric patients.

Prehospital emergency care of children is already challenging. Life-threatening pediatric emergencies are low-frequency but high-stakes events. In the United States, paramedics and emergency medical technicians serve as PHPs, but only paramedics perform advanced life support procedures for both adults and children. Preparations for pediatric emergencies require even more attention to ensure the correct selection of appropriately sized equipment and calculation of weight-based medication dosages. Because of the relative infrequency of life-threatening emergencies in children, simulation is a useful adjunct for PHPs to practice these skills. This study sought to examine the impact of Level B PPE on the ability of paramedics to perform simulated clinical tasks when compared with regular attire.

\section{METHODS \\ Participants}

This prospective single-arm study of PHPs was performed at the simulation center of a suburban Level I trauma and tertiary care center; the local institutional review board deemed the study exempt. A total of 59 eligible paramedics from a suburban fire and rescue department were enrolled in the study. Paramedics were eligible for inclusion if they (a) had experience performing the procedures to be studied, (b) had experience wearing PPE, (c) had been in their current role for at least 1 year, and (d) had no contraindication to wearing PPE. Prescreening included a wellness and blood pressure screening on the morning of each session. Paramedics who failed to meet criteria were excluded from participation. Exclusion criteria also included status as an emergency medical services student, reported problems with claustrophobia, or a history of difficulty wearing PPE.

\section{Study Design}

In this single-group, prospective crossover, pre/post study, participants served as their own controls to limit the impact of interpersonal differences in procedural skill. Demographic information collected on each participant included age, gender, height, training/discipline, area of clinical practice, years of clinical experience, prior experience with PPE, and use of corrective lenses.

Each participant attended 2 sessions separated by a minimum of $2 \mathrm{wk}$. During each session, each participant performed the same sets of procedures in the same sequence. During session 1 , participants wore normal paramedic attire, and all procedures were performed using standard nonsterile nitrile gloves. For session 2, participants donned a Level B PPE ensemble typically worn by the county fire department; this ensemble included a (1) Kappler Encapsulated Level B suit (nongas tight-hooded garment), (2) a Scott AP50 4.5.2002 NFPA (National Fire Protection Association)-compliant selfcontained breathing apparatus (SCBA) with facemask and 60-min duration cylinder, (3) Honeywell North Chemical Resistant 4T453 B131/10 (13 mil) gloves, and (4) Tingley TG-82330 hazardous materials boots. The participants performed selected procedures in Guardian Neoprene smooth Level A chemical hazard class gloves $(35 \mathrm{mil})$. The order of gloves used first was randomized. To avoid learning effects in the PPE session when the 2 types of gloves were worn, data only included the gloves that were worn first in the analysis. The study flow is detailed in Figure 1.

\section{Procedures}

The specific procedures were selected based on their importance and ability to be assessed in a simulated setting. In all cases, timing was measured by a member of the study team who was physically present to observe and used a stopwatch. The success of each task was based on the following predetermined criteria:

\section{A. Airway skills}

1. Bag-mask ventilation (BMV): Participants were asked to perform BMV using a 0.5-L self-inflating bag and a preselected appropriately sized mask on the Laerdal SimJunior manikin (Laerdal, Wappingers Falls, NY). Participants were asked to assemble the bag-mask and perform 3 ventilations that were verified by chest rise. Successful completion was defined as when the chest returned to baseline. Participants were given 5 min to complete the task. Direct observation was used as it was found to be more accurate than software feedback during the pilot testing.

2. Endotracheal intubation: Participants were asked to perform direct laryngoscopy using a 4.5 ET tube and a Miller 1 blade, placed in the same positions at the head of the bed for each trial. Participants were instructed to stand at the head of the bed before starting. Successful completion required manikin intubation and ventilation within $3 \mathrm{~min}$. Time to completion was defined as when the chest returned to baseline.

3. Pulse oximetry probe placement: Participants were asked to place a pulse oximeter probe onto the toe of the manikin. Successful completion required placement in less than a minute. Time to completion was measured from the instruction to begin until probe was secured.

\section{B. Circulation Skills}

1. Intraosseous (IO) needle placement: Participants were asked to place an Arrow EZ-IO using an Arrow EZ-IO Power Driver (Teleflex, Morrisville, NC) into the tibia of a Pediatric HAL 
FIGURE 1

\section{Study Design.}

\begin{tabular}{|c|c|}
\hline Minimum o & sessions \\
\hline Session 1: Normal Attire & Session 2: Level B PPE \\
\hline $\begin{array}{c}\text { Station 1: Airway and breathing skills } \\
\text { Bag-mask-ventilation, tracheal intubation, pulse } \\
\text { oximetry probe placement }\end{array}$ & $\begin{array}{l}\text { Station 1: Airway and breathing skills } \\
\text { Bag-mask-ventilation*, tracheal intubation, } \\
\text { pulse oximetry probe placement }\end{array}$ \\
\hline $\begin{array}{c}\text { Station 2: Circulation skills } \\
\text { Intraosseous needle, intramuscular (IM) } \\
\text { injection (syringe), IM autoinjector, tourniquet } \\
\text { placement }\end{array}$ & $\begin{array}{c}\text { Station 2: Circulation skills } \\
\text { Intraosseous needle* }{ }^{*} \text { IM injection (syringe) } \\
\text { IM autoinjector, tourniquet placement }{ }^{*}\end{array}$ \\
\hline $\begin{array}{c}\text { Station 3: Automated external } \\
\text { defibrillator (AED) skills } \\
\text { Pediatric AED skills, pad placement } \\
\text { and defibrillation }\end{array}$ & $\begin{array}{c}\text { Station 3: AED skills } \\
\text { Pediatric AED skills, pad placement } \\
\text { and defibrillation }\end{array}$ \\
\hline
\end{tabular}

\section{Station 1}
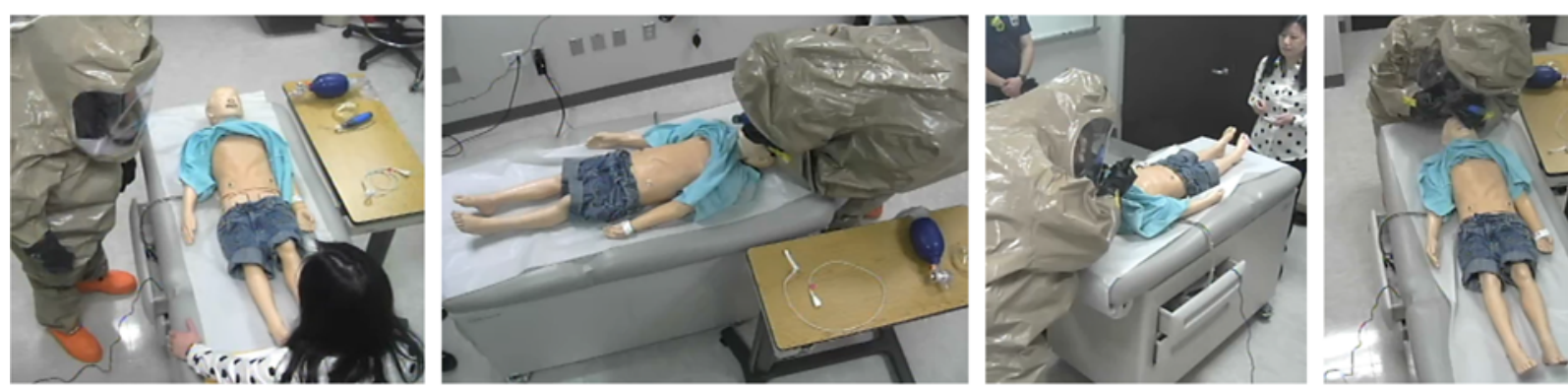

\section{Station 2}
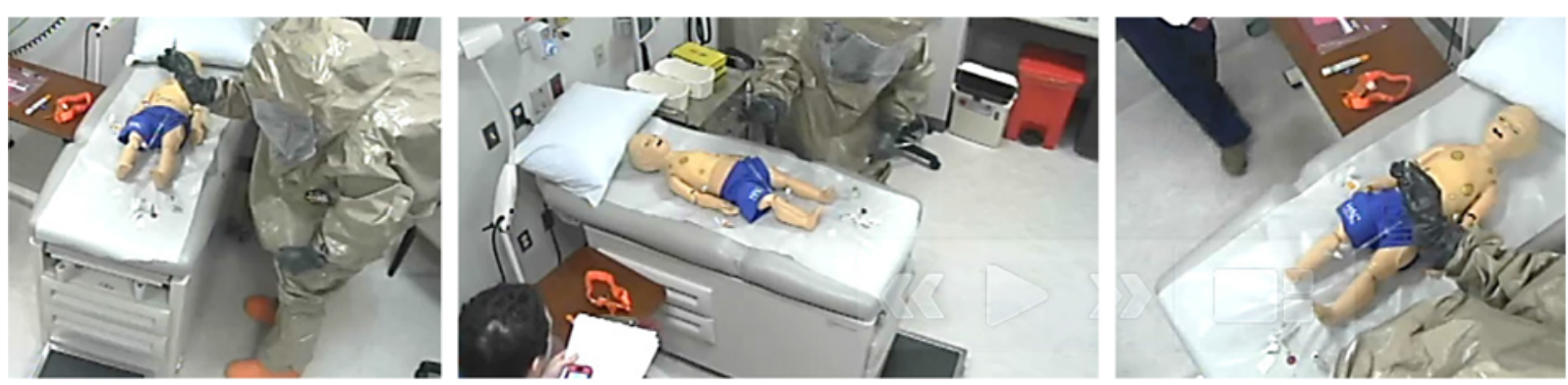

Station 3
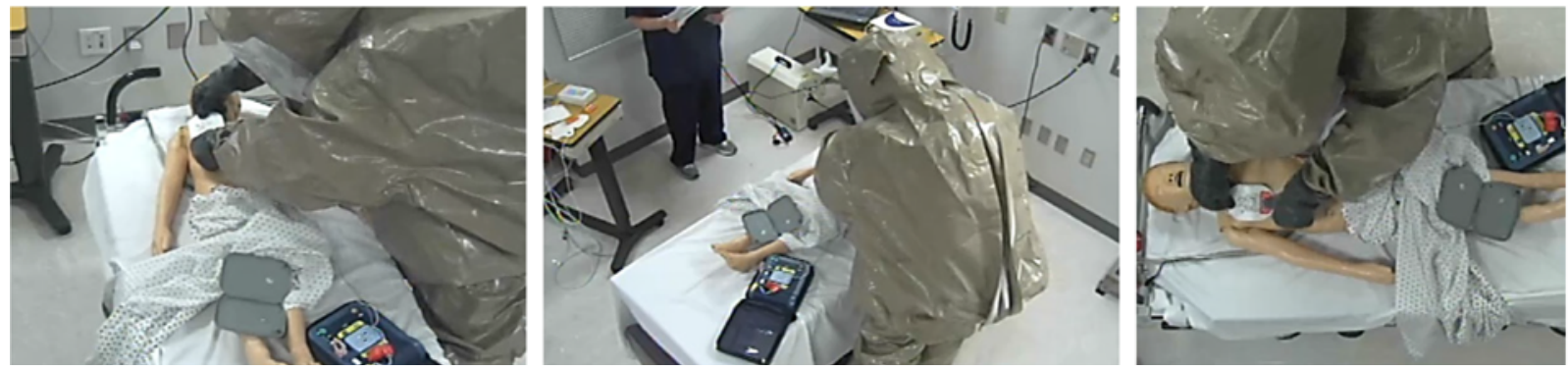
S3004/3004.L One-Year-Old Patient Simulator (Gaumard Scientific, Miami, FL), and attach it to an intravenous (IV) connector. Successful completion was defined as when an IV tubing connector was attached to the IO needle inserted into the tibia in less than $3 \mathrm{~min}$. Time to completion was measured from the instruction to begin until the IV tubing connector was attached to the IO needle inserted in the tibia.

2. Intramuscular (IM) injection: Participants were asked to remove a 5 - $\mathrm{mL}$ syringe and 18 -gauge needle from sterile packaging, assemble the equipment, and draw up $2 \mathrm{~mL}$ of simulated medication from a single-use $10-\mathrm{mL}$ sterile fluid vial. The participant then delivered the injection into the thigh of the manikin. Success was defined as completion of the injection within $2 \mathrm{~min}$. Times to completion were measured from the instruction to begin until (a) the needle was removed from the vial and (b) when the needle was removed from the thigh (to capture the time taken both to prepare the medication and then to administer it).

3. IM autoinjector: Participants used an EpiPEN (Pfizer Canada Inc., Kirkland, Quebec) autoinjector trainer to deliver a simulated dose of epinephrine into the thigh of the manikin and to hold for $10 \mathrm{~s}$. Success was defined as medication delivery within $2 \mathrm{~min}$. Time to completion was measured from the instruction to begin until the needle was removed from the thigh.

4. Tourniquet placement: Participants were asked to place a Combat Application Tourniquet (CAT Resources, Rock Hill, SC) around the thigh of the manikin and perform 2 turns of the windlass clip to lock the tourniquet. Successful completion was when the tourniquet was locked within $3 \mathrm{~min}$. Time to completion was measured from the instruction to begin until the tourniquet was locked.

\section{Automated External Defibrillator Pad Application and Defibrillation}

Participants were asked to place a pediatric adaptor into the automated external defibrillator (AED) device (Philips MRx; Koninklijke Philips N.V., Amsterdam, Netherlands) and then place the adhesive defibrillator pads onto the chest wall of the 5-y-old child manikin (MegaCode Kid, Laerdal, Wappingers Falls, NY). Successful completion was the delivery of a shock. Time was measured from the instruction to begin until the AED noted that a shock was delivered.

The interrater reliability for the primary time to completion measures was assessed through timing of the participant with 2 raters in a sample of visits. Twenty percent of participants were selected, and only PPE visits were used for this assessment.

A multiple-choice attitude survey was administered to participants at both sessions to assess whether (1) nonsterile gloves would interfere with procedures, (2) full-body PPE suits interfered with procedures, (3) PPE made it difficult for the provider to focus upon the procedure, (4) PPE was claustrophobic, (5) the provider would be slower performing procedures in the full-body PPE, and (6) the provider felt prepared to appropriately don Level B PPE. A 5-point anchored rating scale was used.

\section{Analysis}

Demographic and baseline characteristics were summarized with frequencies and percentages for categorical data and means and standard deviations (SDs) for continuous data. The primary outcome was time to procedure completion, comparing regular paramedic attire (baseline) and PPE; the secondary outcome was the attitude toward using PPE as assessed in the attitude survey.

For the primary outcome, the median difference in time of performing selected tasks with and without PPE was examined using Wilcoxon signed-rank tests. Differences between the completion times when paramedics wore Level A or B gloves were also compared using 2 -sample median tests. The survey data were analyzed using paired t-tests comparing baseline and post-PPE session responses. ${ }^{8}$ Interrater reliability was assessed for agreement of raters at the PPE session using the intraclass correlation (ICC). All tests were 2 -sided; $P<0.05$ was considered to be statistically significant. Statistical analyses were performed using SAS software version 9.4 (SAS Institute Inc., Cary, NC).

\section{Sample Size Calculation}

The study was powered to detect a 10-s difference in time to completion between baseline and PPE sessions. The SDs of the differences between baseline and PPE sessions was assumed to be as high as $20.6 \mathrm{~s}^{8}$ with an assumed intraindividual correlation of 0.6 between assessments. Using these assumptions, a sample size of 48 participants was sufficient to provide $>90 \%$ power for time to completion endpoints when assessing difference using 95\% 2-sided confidence intervals. Significance levels used in the power analysis for this exploratory analysis were not adjusted for multiplicity.

\section{RESULTS}

A total of 59 eligible paramedics from a suburban fire and rescue department were enrolled; 50 participants attended the 2 study sessions and were included in the analysis. Participant demographics and reported levels of experience are provided in Table 1. The mean paramedic age was 39.6 (SD 6.9) years, and $94.0 \%$ were male. Almost $90 \%$ had previously worn PPE more than 10 times.

Table 2 summarizes the time to completion of each procedure in the order in which the tasks were performed, as well as the procedures that were performed in the 2 types of gloves. For procedures performed in full Level B PPE with Level B gloves only, the median time to completion increased by $4.5 \mathrm{~s}$ $(P=0.01)$ for tracheal intubation, $4 \mathrm{~s}$ for pulse oximetry probe placement $(P<0.0001), 7 \mathrm{~s}$ for IO insertion $(P<0.0001)$, and 


\section{TABLE 1}

\section{Demographic and Baseline Characteristics}

\begin{tabular}{lc} 
& Participants $(\boldsymbol{N}=\mathbf{5 0})$ \\
Mean age (SD), years & $39.6(6.9)$ \\
Male sex & $47(94.0 \%)$ \\
Number of times PPE worn previously & \\
$1-2$ & $1(2.0 \%)$ \\
$3-5$ & $2(4.0 \%)$ \\
$6-10$ & $3(6.0 \%)$ \\
$>10$ & $44(88.0 \%)$ \\
\hline
\end{tabular}

Abbreviation: PPE, personal protective equipment; SD, standard deviation.

$9.5 \mathrm{~s}$ for AED pad placement and defibrillation $(P=0.01)$ compared with the baseline session with regular nitrile gloves.

In the comparison of Level A and Level B gloves, analysis was performed only on the type of glove worn first to minimize the effect of learning. The completion time between baseline and PPE visits for BMV was $1 \mathrm{~s}$ in both Level $\mathrm{A}(P=0.5)$ and Level $\mathrm{B}(P=0.01)$ glove types. The median time for IM injection preparation (drawing up the medication and removing the needle from the vial) increased $21 \mathrm{~s}$ in Level B gloves but $28.5 \mathrm{~s}$ in Level A gloves from baseline ( $P$ for all $<0.0001$ ). The median time to deliver the IM medication (from drawing up the medication, injecting, and then removing the needle from the thigh) increased $23 \mathrm{~s}$ in Level B gloves $(P=0.0001)$ and $35 \mathrm{~s}$ in Level A gloves $(P=0.0001)$ from baseline. The median time to deliver an injection by means of autoinjector increased by $2 \mathrm{~s}$ in Level $\mathrm{B}$ gloves $(P=0.46)$, and $4 \mathrm{~s}$ in Level A gloves $(P=0.03)$ from the baseline. The median times for tourniquet placement increased by $8.5 \mathrm{~s}$ in Level B gloves, and $12.5 \mathrm{~s}$ in Level A gloves ( $P$ for all $<0.0001)$ from baseline (Table 2 ).

Of note, all participants were able to meet the defined standard for procedures except tracheal intubation and IM autoinjector, for which 3 and 1 participants, respectively, did not meet the standard in PPE. Interrater reliability was high (ICC > 0.86) for all tasks except AED implementation $(\mathrm{ICC}=-0.14)$.

\section{Attitudes Survey Results}

On the presession survey (Table 3), the participants agreed or strongly agreed that PPE would interfere with procedures and slow procedures down, and were neutral on the impact of PPE on their focus. They did not believe that the PPE was claustrophobic and believed they were prepared to don PPE. After the PPE session, the participants were significantly more likely to agree that nonsterile gloves affected procedures (mean change score $=1.7 ; P<0.001)$. They also felt more positively after the PPE session than at baseline regarding their preparedness to don PPE (mean change score $=0.5 ; P=0.01$ ). The change in responses from baseline to post-PPE altered the overall group category from disagree to neutral for the impact of nonsterile gloves on procedural performance.

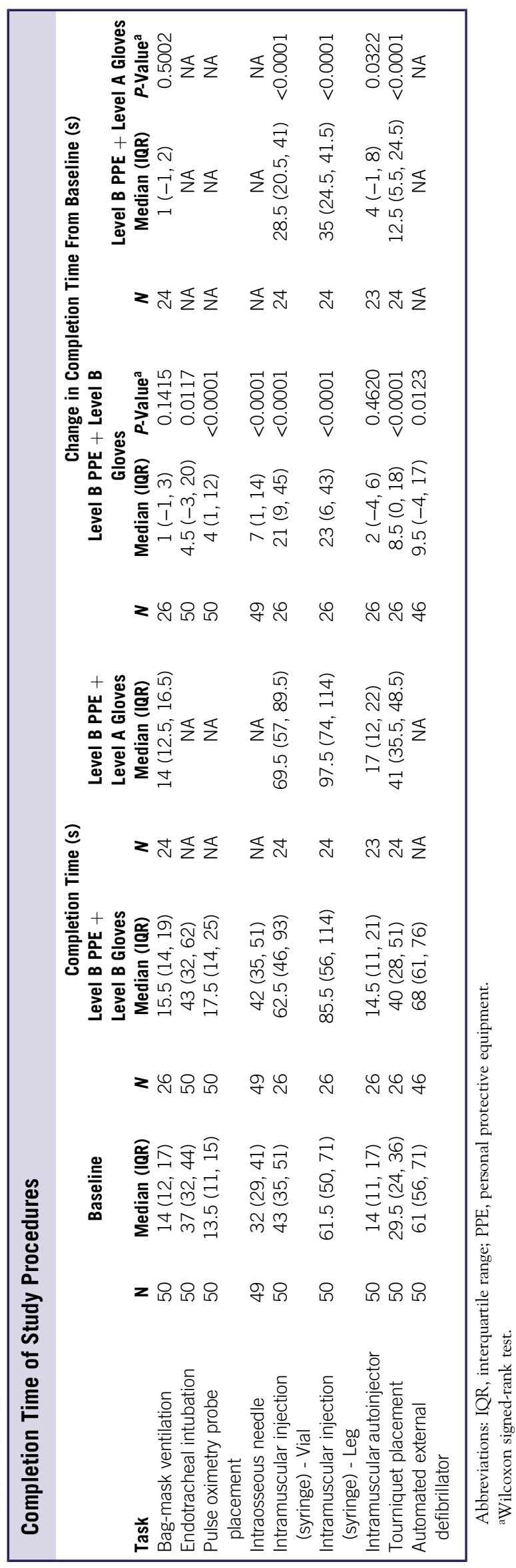

VOL. 16/NO. 1 


\section{Attitude Toward PPE Use}

\begin{tabular}{|c|c|c|c|c|c|c|c|}
\hline \multirow{2}{*}{ Question } & \multicolumn{7}{|c|}{ Attitude Score ${ }^{a}$} \\
\hline & \multicolumn{2}{|c|}{ Baseline } & \multicolumn{2}{|c|}{ PPE } & \multicolumn{3}{|c|}{ Change } \\
\hline $\begin{array}{l}\text { Non-sterile gloves interfere with } \\
\text { procedures }\end{array}$ & 43 & $1.6(1.1)$ & 43 & $3.3(1.2)$ & 43 & $1.7(1.6)$ & $<0.0001$ \\
\hline $\begin{array}{l}\text { Full body PPE suits interfere with } \\
\text { procedures }\end{array}$ & 42 & $4.0(1.1)$ & 42 & $3.9(0.8)$ & 42 & $-0.1(1.3)$ & 0.6430 \\
\hline $\begin{array}{l}\text { PPE makes it hard for me to focus } \\
\text { on my procedure }\end{array}$ & 42 & $3.0(1.2)$ & 42 & $2.6(1.1)$ & 42 & $-0.4(1.6)$ & 0.1357 \\
\hline $\begin{array}{l}\text { Slower performing procedures in } \\
\text { full-body PPE }\end{array}$ & 41 & $4.3(0.8)$ & 41 & $4.0(0.8)$ & 41 & $-0.3(1.1)$ & 0.0794 \\
\hline $\begin{array}{l}\text { Prepared to appropriately don } \\
\text { Level B gear }\end{array}$ & 42 & $4.2(1.3)$ & 42 & $4.7(0.7)$ & 42 & $0.5(1.2)$ & 0.0121 \\
\hline
\end{tabular}

Abbreviations: IQR, interquartile range; $\mathrm{PPE}$, personal protective equipment; SD, standard deviation.

${ }^{\mathrm{a}} 1=$ Strongly Disagree, 2 = Disagree, $3=$ Neither Agree nor Disagree, $4=$ Agree, $5=$ Strongly Agree .

bPaired t-test.

\section{DISCUSSION}

The results revealed that, while Level B PPE did increase the time to completion of some procedures, it had little to no impact on the ability of paramedics to successfully perform all categories of resuscitation procedures in the pediatric model. Our study is the first to address this with PHPs.

\section{Glove Type}

Although glove type was not observed to impact paramedics' performance of BMV or IM autoinjection, the measured completion times of all tasks requiring fine motor skills were longer when performed in Level A gloves, and from baseline (Table 2). This might suggest the thickness of chemical protective gloves $(\mathrm{CPG})$ as a factor in causing delays. After the PPE session, paramedics were also more likely to believe that CPG interfered with procedures than with regular nitrile gloves. Both glove types were observed to interfere with tasks involving handling adhesive material such as tape and AED pads, but actions such as the tearing open of equipment packaging and the twisting of IV connector equipment were observed to be more difficult in CPG. However, differences of $4 \mathrm{~s}$ for tourniquet placement and $12 \mathrm{~s}$ for IM injection when performing these procedures in Level A versus Level B gloves are unlikely to be of clinical significance. This suggests that CPG thickness may not necessarily be the factor that inhibits these skills, but just the CPG themselves.

When comparing times of IM injection, paramedics wearing CPG took up to $35 \mathrm{~s}$ (Level A) and $23 \mathrm{~s}$ (Level B) longer than baseline, which was also statistically significant. While this is also unlikely to be clinically significant, CPG only caused an increase of $4 \mathrm{~s}$ from baseline in IM autoinjection in Level A gloves. A retrospective study ${ }^{9}$ investigating survival after epinephrine administration for nonshockable out-of-hospital arrest in 595 pediatric patients found that the odds of survival were $9 \%$ lower for every minute of delay in administration of epinephrine, with $71 \%$ of children receiving epinephrine by means of intraosseous. The odds ratio of survival when epinephrine was given late versus early was 0.43 . The results of our study support that IM autoinjection is preferable to an IM injection when in CPG. Although not studied here, the insertion of a simple supraglottic device would also probably not be hindered by $\mathrm{CPG}^{10}$ as tracheal intubation was minimally impacted by CPG versus baseline.

To explore other reasons for PPE's impact on procedures, we observed the ability of the paramedics to see or hear instructions in the encapsulating hood and SCBA equipment. Level B PPE was chosen as it closely resembles Level A PPE with minor differences: Level A PPE has built-in gloves and is airtight once the seal is fastened (there are no vents). While Level B PPE is not gas-tight, some participants experienced a significant buildup of condensation on the inside of the hood that impaired visibility and required them to pause between stations to wipe off excessive fog. Although this was not observed to relate to paramedics' levels of physical activity, this could not be measured in this study. The paramedics also moved with more difficulty around obstacles and were observed to need to intermittently move their bodies awkwardly (from an immobile stance) to stop the oxygen tank safety sensor alarm from ringing. The impact of the weight of the gear on paramedics' abilities was not investigated in this study. It should be noted that, although the pre-PPE attitudes survey revealed that paramedics did not believe that PPE would interfere with their ability to perform procedures, they reported feeling more prepared to don Level B PPE following the PPE session.

\section{Limitations}

Variability in the level of provider experience would be a possible source of bias. As the intent of the study was to investigate the impact of the PPE on procedural performance, the study 
population included only experienced paramedics. Eightyeight percent had previously worn Level B PPE at least 10 times. PHPs with less training or pediatric experience or with no prior experience in PPE might have encountered additional barriers that were not noted in this study. These results may not be generalizable to all PHPs. We also acknowledge the study group was mostly male, a reflection of the proportion of PHPs identifying as female in the workforce; thus, the study was not powered to investigate gender-based impact on performance.

We also note that realism is often an aspect of simulation that affects environmental fidelity. ${ }^{11}$ The decreased ability of the paramedics to see or hear and limited mobility in PPE might be reproduced in a real disaster environment, but a pediatric manikin may not be a good substitute for a pediatric patient. A mobile, injured, combative, wet, or cold child would probably resist care from a provider dressed in PPE without assistance from a familiar caretaker, further complicating the environment.

To standardize the study design across 3 study sites and sessions, each procedural task was simplified and performed with brand new equipment. Manikins were placed on stretchers at waist height. To remove the effect of retrieving equipment from a bag, it was laid out on countertops for easy access. The rooms were air-conditioned with ample space to move about. For safety reasons, a volunteer assisted each participant with donning and doffing, and cleaning off their visor. In realworld encounters and unpredictable environments, the paramedics would not have a "spotter" or be able to clean their own visor. It is possible that prolonged crouched or awkward positions in PPE could complicate procedural performance (eg, "on the floor" intubation ${ }^{12}$ ) or increase metabolic demand. ${ }^{13}$ The latter could not be studied due to the oxygen tank supply and our participants were not in PPE for greater than $1 \mathrm{~h}$. Given current conditions where providers are in PPE for prolonged periods of time, this would be useful to investigate. Last, the integrity or durability of the suit could be theoretically affected by kneeling on rough surfaces, but this was not identified in the literature.

It could be argued that these procedures would not be undertaken in a prehospital response to a large-scale disaster involving a biohazard. The JumpSTART pediatric triage algorithm ${ }^{14}$ and operations plans for Hot Zone responses ${ }^{15}$ recommend the prioritization of resuscitation efforts when faced with multiple critical patients. This study supports that in the case of a single pediatric patient suffering from exposure to a biohazard, airway rescue, or autoinjection of antidote/pressor by an experienced provider would not be impacted by wearing Level B PPE.

This simulation study of a pediatric model adds to the literature to address the assumption that Level B PPE would impact the performance of resuscitative procedures by PHPs. However, it is still imperative that PHPs have access to pediatric procedural practice sessions while in PPE. At the very least, procedural training in CPG could be useful where PPE is a commodity or in scarce supply.

\section{CONCLUSIONS}

The use of Level B PPE made no statistically significant impact on experienced paramedics' measured performance of BMV and IM autoinjection in a pediatric model and suggests autoinjection is preferable for resuscitation medication administration when using CPG. While there was no clinically significant impact on the paramedics' overall completion of the remainder of advanced resuscitative procedures, this study suggests that pediatric preparedness and training drills in Level B PPE would especially be of benefit for PHPs with less experience. Preparedness to work in Level B PPE should continue to be emphasized, especially when the use of CPG is required, and is not a deterrent to resuscitation attempts on a pediatric patient in the right clinical scenario. This information provides guidance to those preparing for events requiring Level B PPE in the care of acutely ill children.

\section{About the Authors}

Department of Emergency Medicine, Inova Fairfax Medical Campus, Falls Church, Virginia (Drs Kou, Kochman); Inova Center for Advanced Medical Simulation, Inova Fairfax Medical Campus, Falls Church, Virginia (Drs Kou, Stacks, Ms Semiao); Departments of Anesthesia $\mathbb{E}$ Critical Care Medicine and Pediatrics, Perelman School of Medicine at the University of Pennsylvania, Philadelphia, Pennsylvania (Dr Donoghue); Fairfax County Fire and Rescue Department, Hazardous Materials Response Team, Fairfax, Virginia ( $\mathrm{Mr}$ Nash); Eunice Kennedy Shriver National Institute of Child Health and Human Development, National Institutes of Health, Bethesda, Maryland (Dr Siegel); Duke Clinical Research Institute, Duke University School of Medicine, Durham, North Carolina (Dr Ku, Ms Gosnell); The Emmes Company, Rockville, Maryland (Ms Debski, Drs Chen, Sharma); Department of Pediatrics, Feinberg School of Medicine, Northwestern University, Chicago, Illinois (Drs Krug, Adler); Department of Medical Education, Feinberg School of Medicine, Northwestern University, Chicago, Illinois (Dr Adler) and H. Stacks is now at George Mason University, Fairfax, Virginia.

Correspondence and reprint requests to Maybelle Kou, Inova Fairfax Medical Center/Inova Childrens' Hospital, 3300 Gallows Road, Falls Church, VA 22042 (e-mail: maybelle.kou@inova.org).

\section{Acknowledgments}

The researchers thank Inova Center for Advanced Medical Simulation technicians Mathew Huddleston and Krzysztof Bieniek for their many hours of assistance with preparation and data collection, as well as Dr. Dan Ochsenschlager, the fellows of the Altieri Pediatric Emergency Medicine Fellowship, and Fairfax County Fire and Rescue support staff at the study sessions. The group is especially grateful to Peter Hoffmann of the Duke Clinical Research Institute for his dedication and support to the writing team.

\section{Funding}

This work was funded under National Institute of Child Health and Human Development (NICHD) contract HHSN275201000003I for the Pediatric Trials Network (principal investigator, Danny Benjamin). The content is solely the responsibility of the authors and does not necessarily represent the official views of the National Institutes of Health (NIH). 


\section{Pediatric Trials Network (PTN) Steering Committee Members}

Daniel K. Benjamin Jr., Christoph Hornik, Kanecia Zimmerman, Phyllis Kennel, and Rose Beci, Duke Clinical Research Institute, Durham, NC; Chi Dang Hornik, Duke University Medical Center, Durham, NC; Gregory L. Kearns, Independent; Matthew Laughon, University of North Carolina at Chapel Hill, Chapel Hill, NC; Ian M. Paul, Penn State College of Medicine, Hershey, PA; Janice Sullivan, University of Louisville, Louisville, KY; Kelly Wade, Children's Hospital of Philadelphia, Philadelphia, PA; Paula Delmore, Wichita Medical Research and Education Foundation, Wichita, KS.

\section{The Eunice Kennedy Shriver National Institute of Child Health and Human Development (NICHD) \\ Perdita Taylor-Zapata and June Lee.}

\section{The Emmes Company, LLC (Data Coordinating Center)}

Ravinder Anand, Gaurav Sharma, Gina Simone, Kim Kaneshige, and Lawrence Taylor.

\section{PTN Publications Committee}

Chaired by Thomas Green, Ann \& Robert H. Lurie Children's Hospital of Chicago, Chicago, IL.

\section{Authors' Contributions}

M.K.: study design, data collection, analysis and interpretation, drafting and revisions of the manuscript for important intellectual content and statistical expertise; A.J.D. and M.D.A.: study design, analysis and interpretation, drafting and manuscript revisions; S.K. and D.S.: study design, interpretation, acquisition of funding; M.N.: study design, data collection and interpretation of data; H.S., A.K., and M.S.: data collection, revisions; L.K. and J.D.: study design, manuscript revisions; J.C. and G.S.: statistical expertise.

\section{Conflicts of Interest}

None of the authors have any relevant conflicts of interest.

\section{REFERENCES}

1. Centers for Disease Control and Prevention. Infection prevention and control recommendations for hospitalized patients under investigation (PUIs) for Ebola virus disease (EVD) in U.S. hospitals. https://www.cdc. gov/vhf/ebola/healthcare-us/hospitals/infection-control.html. Accessed September 17, 2018.

2. World Health Organization. Interim infection prevention and control guidance for care of patients with suspected or confirmed Filovirus haemorrhagic fever in health care settings, with focus on Ebola. September 2014. http://www.euro.who.int/_data/assets/pdf_file/0005/268772/InterimInfection-Prevention-and-Control-Guidance-for-Care-of-Patients-withSuspected-or-Confirmed-Filovirus-Haemorrhagic-Fever-in-Health-CareSettings,-with-Focus-on-Ebola-Eng.pdf. Accessed September 17, 2018.

3. Occupational Safety and Health Administration. OSHA best practices for hospital-based first receivers of victims from mass casualty incidents involving the release of hazardous substances. January 2005. Table 3. https://www.osha.gov/dts/osta/bestpractices/html/hospital_firstreceivers. html\#appa213. Accessed March 14, 2020.

4. US Department of Health and Human Services. Chemical hazards emergency medical management. Personal protective equipment. https://chemm. nlm.nih.gov/ppe.htm. Accessed March 14, 2020.

5. Coates MJ, Jundi AS, James MR. Chemical protective clothing; a study into the ability of staff to perform lifesaving procedures. J Accid Emerg Med. 2000;17:115-118.

6. Chen J, Lu KZ, Yi B, et al. Chest compression with personal protective equipment during cardiopulmonary resuscitation: a randomized crossover simulation study. Medicine (Baltimore). 2016;95:e3262.

7. $\mathrm{Kim} \mathrm{TH}, \mathrm{Kim} \mathrm{CH}$, Shin SD, et al. Influence of personal protective equipment on the performance of life-saving interventions by emergency medical service personnel. Simulation. 2016;92:893-898.

8. Rinderknecht AS, Mittiga MR, Meinzen-Derr J, et al. Factors associated with oxyhemoglobin desaturation during rapid sequence intubation in a pediatric emergency department: findings from multivariable analyses of video review data. Acad Emerg Med. 2015;22:431-440.

9. Hansen M, Schmicker RH, Newgard CD, et al. Time to epinephrine administration and survival from nonshockable out-of-hospital cardiac arrest among children and adults. Circulation. 2018;137:2032-2040.

10. Castle N, Pillay Y, Spencer N. Comparison of six different intubation aids for use while wearing CBRN-PPE: a manikin study. Resuscitation. 2011; 82:1548-1552.

11. Cheng A, Nadkarni VM, Mancini MB, et al. Resuscitation education science: educational strategies to improve outcomes from cardiac arrest: a scientific statement from the American Heart Association. Circulation. 2018;138:e82-e122.

12. Castle N, Owen R, Clarke S, et al. Does position of the patient adversely affect successful intubation whilst wearing CBRN-PPE? Resuscitation. 2010;81:1166-1171.

13. Stein C, Makkink A, Vincent-Lambert C. The effect of physical exertion in chemical and biological personal protective equipment on physiological function and reaction time. Prehosp Emerg Care. 2010;14:36-44.

14. Romig LE. Pediatric triage. A system to JumpSTART your triage of young patients at MCIs. JEMS. 2002;27(7):52-58, 60-63.

15. Federal Emergency Management Agency. Planning guidance for response to a nuclear detonation, 2nd edition. June 2010. https:/www.fema.gov/ media-library-data/20130726-1821-25045-3023/planning_guidance_for_ response_to_a_nuclear_detonation_2nd_edition_final.pdf. Accessed September $18,2018$. 